\title{
MicroRNA-modulated targeting of vascular smooth muscle cells
}

\author{
Michael S. Parmacek \\ University of Pennsylvania Cardiovascular Institute and Department of Medicine, Philadelphia, Pennsylvania, USA.
}

\begin{abstract}
VSMCs exhibit the remarkable plasticity required for development and adaptation of the cardiovascular system. The capacity of VSMCs to modulate their phenotype has evolved to facilitate angiogenesis and wound healing, but it has also been implicated in the pathogenesis of atherosclerosis, restenosis, posttransplant arteriopathy, and pulmonary hypertension. In this issue of the JCI, Boettger and colleagues report that the recently discovered Mir143/145 gene cluster promotes acquisition of the contractile phenotype of murine VSMCs (see the related article beginning on page 2634). These VSMC-restricted microRNAs, which target unique combinations of SMC genes, provide an efficient mechanism to fine-tune cardiovascular homeostasis and the response of the vessel wall to injury. This important discovery will open the door to new avenues of investigation and potentially future therapies for vascular diseases.
\end{abstract}

VSMCs regulate arterial tone and blood pressure through the expression of a unique set of genes encoding SMC lineage-restricted myofibrillar and cytoskeletal proteins and cell-surface receptors. In contrast to the striated muscle cell lineages, SMCs retain the capacity to proliferate and reversibly modulate their phenotype during postnatal development (reviewed in ref. 1). In response to vascular injury and the release of growth factors including PDGF, TGF- $\beta$, and Ang II, VSMCs modulate their phenotype from a contractile SMC, expressing high levels of contractile and cytoskeletal proteins, to a proliferative, synthetic SMC, expressing abundant cytokines and extracellular matrix (reviewed in ref. 1). The modulation of vascular SMC phenotype has been implicated in the pathogenesis of vascular proliferative syndromes including atherosclerosis, restenosis following angioplasty, and transplant arteriopathy (reviewed in refs. 1, 2). Remarkably, VSMCs arise from three distinct origins in the embryo: the lateral mesoderm, which gives rise to vascular and visceral SMCs; the cardiac neural crest, which gives rise to SMCs populating the cardiac outflow tract and great arteries; and the proepicardial organ, which gives rise to coronary artery SMCs (reviewed in ref. 3). The complex lineage relationships and lack

Conflict of interest: The author has declared that no conflict of interest exists.

Citation for this article: J. Clin. Invest. 119:2526-2528 (2009). doi:10.1172/JCI40503. of definitive, and stable, SMC markers have complicated assessment of the molecular mechanisms regulating specification and differentiation of the SMC lineage and modulation of VSMC phenotype in response to arterial injury.

Multiple studies performed over the last decade have revealed that a transcriptional complex built around the MADS box transcription factor, serum response factor (SRF), and the SMC- and cardiomyocyterestricted transcriptional coactivator myocardin plays a central role in regulating SMC differentiation and promoting the contractile SMC phenotype (reviewed in ref. 4). Functionally important SRF-binding sites, or CArG boxes, have been identified in transcriptional regulatory elements controlling expression of multiple genes encoding SMC-restricted contractile and cytoskeletal proteins (5). Forced expression of myocardin in ES cells activates most, but not all, genes associated with the contractile SMC phenotype $(6,7)$. Myocardin directly influences the chromatin structure required for activation of genes encoding SMC contractile proteins $(8,9)$. Mice harboring a null mutation in the myocardin gene (Myocd) exhibit profound defects in the yolk sac and embryonic vasculature, most likely attributable to a block in SMC differentiation (10). Myocd-deficient VSMCs exhibit ultrastructural and biochemical features generally associated with the SMC synthetic phenotype, including loss of contractile proteins and increased synthesis of extracellular matrix (11). SRF integrates multiple signals influencing SMC phenotype via its capacity to differentially associate with myocardin and ternary complex factors (TCFs) in the Ets family of transcription factors, including Elk-1 (12). In addition, a myriad of other intracellular signaling pathways converge upon SRF and myocardin in a combinatorial fashion and together modulate SMC phenotype (4).

\section{The Mir143/145 gene cluster promotes the contractile SMC phenotype}

In this issue of the JCI, Boettger and colleagues describe mice harboring a null mutation in the newly discovered Mir143/145 gene cluster, revealing an unanticipated role of these SMC-enriched microRNAs (miRNAs) in the regulation of VSMC phenotype (13). This study complements a recent article by Cordes et al. that reported that miR-145 and miR-143 regulate SMC fate and plasticity (14). Both studies present compelling data showing that SMC phenotype and differentiation are regulated, at least in part, by SMC-restricted miRNA species. miRNAs are small, untranslated RNA species (20-25 nucleotides) that have been implicated in the regulation of gene expression essential for cellular differentiation, proliferation, homeostasis, and organ development (15). They function either by targeting specific mRNA species for degradation or by translational interference (15). Using miRNA target prediction algorithms, hundreds of putative target mRNAs may be identified, although target gene prediction is an evolving science that still requires rigorous experimental validation $(15,16)$. While both studies $(13,14)$ highlight the importance of the Mir143/145 gene cluster in the SMC lineage, the function(s) attributed to miR-143 and miR-145 in SMCs differ in several fundamental respects that merit careful consideration as the field moves forward.

The Mir143/145 gene cluster includes Mir143 and Mir145, which lie within a 1.7-kb highly conserved region of mouse chromo- 
some $18(13,14)$. Boettger and colleagues performed a series of miRNA microarray hybridization experiments revealing that miR-143 and miR-145 are enriched in murine SMCs (13). Using complementary genetic approaches, both groups observed that, in the mouse embryo, miR-143/145 expression is restricted to heart, vascular, and visceral SMCs $(13,14)$. During late fetal and postnatal development, miR-143/145 expression is downregulated in the heart but persists in vascular and visceral SMCs. Cordes and colleagues discovered a transcriptional enhancer that is necessary and sufficient to recapitulate the temporal and spatial pattern of miR-143/145 expression in the embryo (14). Mutation of a conserved SRF-binding site abolished activity of the Mir143/145 enhancer in SMCs, suggesting that the Mir143/145 gene cluster may lie downstream of SRF and/or myocardin in the developmental program regulating SMC differentiation. Further studies examining the mechanisms regulating transcription of the Mir143/145 gene cluster are warranted, as miR-143 is enriched during differentiation of mouse ES cells into cardiomyocyte progenitors, and a putative miR-145 target sequence was identified in the Kruppel-like factor 4 (Klf4) gene, which encodes a transcription factor associated with pluripotency $(14,17,18)$.

Boettger and colleagues generated genetically engineered mice in which the Mir143/145 gene cluster was replaced by a lac $Z$ reporter gene and rendered functionally null (13). Homozygous Mir143/145 mutant mice were viable but exhibited thinning of the arterial tunica media (muscular layer), with a reduction in the number of contractile SMCs and a concomitant increase in the number of synthetic SMCs. By contrast, airway function and gastrointestinal motility were unaffected in Mir143/145 mutant mice, suggesting strongly that the Mir143/145 gene cluster selectively regulates vascular versus visceral SMCs in vivo. Physiological characterization of Mir143/145 mutant mice and arterial segments revealed defects in agonist-induced SMC contractility and homeostatic control of blood pressure. Combined genomic/proteomic and transcriptional analyses identified multiple potential miR-143/145 targets including angiotensin I-converting enzyme 1 (Ace). In this regard, it is noteworthy that Ang II, a product of ACE-mediated cleavage of Ang I, is bound by the Ang II type $1\left(\mathrm{AT}_{1}\right)$ receptor on the surface of VSMCs, promoting vasoconstriction and alteration of VSMC phenotype (19); this provides one potential explanation for selective targeting of the vascular system in these mutant mice. Consistent with this observation, pharmacological inhibition of $\mathrm{ACE}$ or the $\mathrm{AT}_{1}$ receptor partially reversed vascular dysfunction and normalized gene expression in the mutant mice. However, the precise mechanism of miR-143/145-induced alterations in blood pressure remains to be elucidated and most likely involves multiple miR-143/145-regulated targets in addition to ACE. This is consistent with the general mechanism of action of miRNAs, which exert their effects by targeting multiple mRNA species often involved in a common pathway. Nevertheless, these data raise the intriguing possibility that miR-143 and miR-145 may influence homeostatic control of blood pressure. Moreover, the observation of spontaneously arising neointimal lesions in normolipidemic miR-143/145 mutant mice provides new insights into the pathogenesis of atherosclerosis (13).

\section{Mir143/145 and SMC fate}

Cordes and colleagues propose that miR-145 and miR-143 regulate SMC fate and plasticity (14). Their conclusions are based primarily upon in vitro experiments demonstrating that antisense oligonucleotides that block miR-145 (antagomirs) inhibit myocardin-induced "reprogramming" of murine $10 \mathrm{~T} 1 / 2$ fibroblasts to the SMC fate and repress a subset of genes encoding SMC-contractile proteins (14). In addition, they observed that miR-145 enhanced myocardin-induced reprogramming of 10T1/2 fibroblasts to SMCs. Of note, forced expression of miR-143 or miR-145, alone or in combination, was not sufficient to reprogram fibroblasts to SMCs in the absence of myocardin. However, miR-145 enhanced TGF- $\beta$-induced differentiation of Joma 1.3 undifferentiated neural crest cells into SMCs in vitro. Putative binding sites for miR-145 were identified in the Myocd and Klf4 3' UTRs, and a putative miR-143-binding site was identified in the 3' UTR of the Elk1 mRNA. Surprisingly, miR-145 relieved transcriptional repression of a reporter plasmid containing the putative Myocd target sequence but enhanced transcriptional repression of a reporter plasmid containing the Klf4 target sequence. Based upon these findings, Cordes and colleagues proposed a binary working model whereby miR-145 promotes myocardin binding to SRF, activating transcription of SMC contractile/ differentiation genes, while miR-143 and miR-145 repress Elk1 and Klf4, transcription factors that enhance SMC proliferation and synthetic functions (12).

While these data suggest that miR-143 and miR-145 regulate SMC fate and plasticity, analyses of Mir143/145 mutant mice, as reported by Boettger et al. in this issue of the JCI (13), argue strongly that these conclusions should be qualified and reconsidered. The demonstration of vascular and visceral SMCs in Mir143/145-null mutant mice demonstrates unequivocally that miR-143 and/or miR-145 are not required for specification of vascular and visceral SMCs in vivo. This apparent discrepancy reflects limitations of the in vitro methodologies that fail to fully recapitulate the biological context captured by gene targeting and in vivo models. One possible explanation is that a myocardin-independent SMC differentiation program may exist (20). In addition, while both studies demonstrate that the Mir143/145 gene cluster promotes acquisition of the SMC phenotype, the results of combined genomic/ proteomic analysis of Mir143/145 mutant aortas argue strongly against a simple binary model whereby miR-143 and/or miR-145 selectively activate SMC contractile proteins while repressing SMC synthetic proteins. Instead, the unique subset of genes and proteins identified by genomic/proteomic analyses of aortas harvested from Mir143/145 mutant mice support a molecular model whereby miR-143 and/or miR-145 regulate a more selective, or nuanced, response of the vessel wall to injury involving unique combinations of downstream targets that influence vascular SMC contractility and phenotype (13).

Irrespective of their differences, these two studies $(13,14)$ serve to identify a critical role of the Mir143/145 gene cluster in the SMC lineage. Because miR-143 and miR-145 are expressed in an SMC-restricted fashion and in theory target multiple SMC genes, they provide an efficient mechanism to fine-tune and coordinate arterial response to vessel wall injury beyond firstorder transcriptional regulation. However, these exciting studies raise as many questions as they answer. What mechanisms regulate expression and activity of miR-143 and miR-145 in SMCs? Do miR-143 and miR-145 regulate common, overlapping, or distinct functions? Given, the unique nucleotide sequences of miR-143 and miR-145, it is likely that they bind to, and target, unique sets of genes in VSMCs. How does the 
Mir143/145 gene cluster alter homeostatic control of blood pressure, and are miRNAs involved in pathological conditions including atherosclerosis? The answers to these questions promise to provide new insights into our understanding of cardiovascular development and the pathogenesis of vascular proliferative syndromes.

\section{Acknowledgments}

This commentary was supported in part by NIH grants R01 HL094520 and P01 HL075215 and the Commonwealth of Pennsylvania.

Address correspondence to: Michael S. Parmacek, University of Pennsylvania School of Medicine, 9035 Gates Pavilion, 3400 Spruce St., Philadelphia, Pennsylvania 19104, USA. Phone: (215) 662-3140; Fax: (215)349-8017;E-mail: michael.parmacek@ uphs.upenn.edu.

1. Owens, G.K., Kumar, M.S., and Wamhoff, B.R 2004. Molecular regulation of vascular smooth muscle cell differentiation in development and disease. Physiol. Rev. 84:767-801.
2. Schwartz, S.M. 1997. Smooth muscle migration in atherosclerosis and restenosis. J. Clin. Invest. 100:S87-S89.

3. Majesky, M.W. 2009. Developmental biology in the vasculature - review series. Arterioscler. Thromb. Vasc. Biol. 29:622.

4. Parmacek, M.S. 2007. Myocardin-related transcription factors: critical coactivators regulating cardiovascular development and adaptation. Circ. Res. 100:633-644.

5. Sun,Q.,etal.2006. Definingthemammalian CArGome. Genome Res. 16:197-207.

6. Du, K.L., et al. 2003. Myocardin is a critical serum response factor cofactor in the transcriptional program regulating smooth muscle cell differentiation. Mol. Cell. Biol. 23:2425-2437.

7. Yoshida, T., Kawai-Kowase, K., and Owens, G.K. 2004. Forced expression of myocardin is not sufficient for induction of smooth muscle differentiation in multipotential embryonic cells. Arterioscler. Thromb. Vasc. Biol. 24:1596-1601.

8. McDonald, O.G., Wamhoff, B.R., Hoofnagle, M.H., and Owens, G.K. 2006. Control of SRF binding to CArG box chromatin regulates smooth muscle gene expression in vivo. J. Clin. Invest. 116:36-48.

9. Zhou, J., et al. 2009. The SWI/SNF chromatin remodeling complex regulates myocardin-induced smooth muscle-specific gene expression. Arterioscler. Thromb. Vasc. Biol. 29:921-928.

10. Li, S., Wang, D.Z., Wang, Z., Richardson, J.A., and Olson, E.N. 2003. The serum response factor coactivator myocardin is required for vascular smooth muscle development. Proc. Natl. Acad. Sci. U. S. A. 100:9366-9370.
11. Huang, J., et al. 2008. Myocardin regulates expression of contractile genes in smooth muscle cells and is required for closure of the ductus arteriosus in mice. J. Clin. Invest. 118:515-525.

12. Long, X., Creemers, E.E., Wang, D.Z., Olson, E.N., and Miano, J.M. 2007. Myocardin is a bifunctional switch for smooth versus skeletal muscle differentiation. Proc. Natl. Acad. Sci. U. S. A. 104:16570-16575.

13. Boettger, T., et al. 2009. Acquisition of the contractile phenotype by murine arterial smooth muscle cells depends on the Mir143/145 gene cluster. J. Clin. Invest. 119:2634-2647.

14. Cordes, K.R., et al. 2009. miR-145 and miR-143 regulate smooth muscle cell fate and plasticity. Nature. Online publication ahead of print. doi:10.1038/nature08195

15. Bartel, D.P. 2009. MicroRNAs: target recognition and regulatory functions. Cell. 136:215-233.

16. Rajewsky, N. 2006. microRNA target predictions in animals. Nat. Genet. 38(Suppl.):S8-S13.

17. Ivey, K.N., et al. 2008. MicroRNA regulation of cell lineages in mouse and human embryonic stem cells. Cell Stem Cell. 2:219-229.

18. Takahashi, K., et al. 2007. Induction of pluripotent stem cells from adult human fibroblasts by defined factors. Cell. 131:861-872.

19. Schieffer, B., et al. 2000. Expression of angiotensin II and interleukin 6 in human coronary atherosclerotic plaques: potential implications for inflammation and plaque instability. Circulation. 101:1372-1378

20. Pipes, G.C., et al. 2005. Stem cells and their derivatives can bypass the requirement of myocardin for smooth muscle gene expression. Dev. Biol. 288:502-513.

\title{
Defining a role for the homeoprotein Six1 in EMT and mammary tumorigenesis
}

\author{
Derek C. Radisky
}

\author{
Department of Cancer Biology, Mayo Clinic, Jacksonville, Florida, USA.
}

\begin{abstract}
Homeobox (Hox) genes encode transcription factors that act as critical regulators of growth and differentiation during embryogenesis. While many studies have identified increased expression of Hox genes in tumors, much less is known about the mechanistic basis by which Hox genes facilitate tumor development. In this issue of the JCI, McCoy and colleagues show that transgenic mice that express the homeoprotein Six 1 in mammary epithelial cells show increases in stem/progenitor cell populations and subsequent tumor development, while in a separate study Micalizzi and colleagues show that overexpression of $\mathrm{Six} 1$ facilitates breast cancer cell metastasis by inducing epithelial-mesenchymal transition (EMT) (see the related articles beginning on pages 2663 and 2678, respectively). Their findings implicate Six1 as a central mediator of breast cancer development.
\end{abstract}

Breast cancer progression is a highly orchestrated process that is much more than just acquisition of increased cellular proliferative capacity. The first step in the development of breast cancer, proliferation

Conflict of interest: The author has declared that no conflict of interest exists.

Citation for this article: J. Clin. Invest. 119:2528-2531 (2009). doi:10.1172/JCI40555. of epithelial cells into the central lumen, involves the acquisition by the cancer cells of the ability to evade structural and biochemical constraints imposed by cell-cell and cell-extracellular matrix interactions. Progression from early, proliferative disease through ductal carcinoma in situ to invasive breast cancer requires the ability to break down the basement membrane and to interact with the stromal extracellular matrix. The ultimate development of metastatic capability, the most deadly and least treatable stage of breast cancer progression, requires the cancer cells to separate from the parental tumor mass, to intravasate into and subsequently extravasate from the vasculature, and to settle and grow at a distant site. All of these steps, from initial breakdown of tissue structure, through increased invasiveness, and ultimate distribution throughout the body, are characteristic features of epithelial-mesenchymal transition (EMT), a developmental process in which adhesive epithelial cells acquire the motile and migratory characteristics of mesenchymal cells (1). Both developmental and pathologic EMT are associated with dramatic alterations in patterns of gene expression, and recent studies have shown that a number of the transcription factors that regulate developmental EMT, including Snail, Slug, and Twist, are also activated during breast cancer progression (2). 